\title{
Think Biology from Chemistry View Point
}

\section{Samit Guha*}

Department of Chemistry, Jadavpur University, India

*Corresponding author: Samit Guha, Department of Chemistry, Jadavpur University, Kolkata 700032, India, Email: samitfsu@gmail.com

\section{Editorial}

Volume 2 Issue 4

Received Date: November 27, 2018

Published Date: December 05, 2018

DOI: $10.23880 /$ macij- 16000128

\section{Editorial}

Biology is very much connected to chemistry. The origin of life started because of chemicals. Chemistry may hold a significant role to resolve the present and future challenges against lethal diseases. It is our dream to make a disease-free world and lengthen our life in a healthy manner. The proper awareness about chemical sciences may guide us to live a superior quality of life. It is very difficult to explicate most of the complex biological phenomenon without proper understanding of chemistry. The biological cell membrane is enormously complicated in nature and made of a variety of molecules including lipids, proteins, and carbohydrates. Glycoprotein and glycolipid are expressed on the exterior of cell membrane, which are the potential targeting ligands for various receptors available in other cells. Sensors for cell membrane are imperative kit to elucidate cell functions including cell signaling, communication, and metabolism [1]. Molecular recognition at the cell membrane offer great insights into biological and pathological processes, including tumour growth and metastasis. This is an extremely demanding and elegant problem in chemistry because the biological membrane is a complicated dynamic assembly.

The biological receptors usually depend on noncovalent interactions such as hydrogen bonding, hydrophobic effect, $\pi-\pi$ interactions, anion $-\pi$ interactions, cation- $\pi$ interactions, electrostatic interactions, and van der Waals forces for binding, sensing, signaling, and transportation. In living systems, nucleic acids and enzymes are very choosy for specific nucleotides. These biopolymers have binding site that form noncovalent interactions with the nucleotide. Molecular recognition of amino acids and peptides are fundamental in many biochemical and medicinal processes. The neurotransmitters glutamate and $\gamma$-aminobutyric acid (GABA) interact with transmembrane (TM) receptors such as G protein-coupled receptor through noncovalent bond. This neurotransmitter-TM receptor interaction induces alteration in receptor conformation and its biological activity with succeeding signaling pathways. Malfunction of signaling pathways can lead to fatal diseases. Ligand-receptor binding such as antigenantibody, RGD-integrin, carbohydrate-lectin etc., have numerous prospective applications [2].

Natural ion channels are large complex proteins that span lipid membranes and transport ions in and out of cells. Ion channels are crucial for organisms and its dysfunction cause diseases such as deadly cystic fibrosis in which the function of natural chloride ion channels are interrupted [3]. Synthetic chloride ion channels may be a promising strategy in 'channel replacement therapy' for the treatment of cystic fibrosis. It is also very important to construct drug molecules to block specific ion channel for the treatment of various disorders. Ions are essential for life and play a critical role to regulate and control heart function, nerve and muscle function, body fluids, blood pressure, osmotic balance, metabolism, and signaling. Developments of highly selective ion sensors in cellular environment are required.

There are considerable structural varieties in membrane proteins. However, membrane proteins have a common pattern in the hydrophobic regions, which helps it to span the lipid bilayer. Noncovalent interactions of TM helices are critical for folding of membrane proteins, channel activities, and TM signaling. Protein-protein interactions are indispensable for many biological processes, including cell proliferation, differentiation, signal transduction etc. Proper understanding of noncovalent interactions in protein-protein assemblies may provide a great insight into the design of synthetic receptors for protein surfaces.

Peptides are the foremost compounds in numerous drug design programs. Development of immuno stimulator peptides, peptide based vaccines, peptide 


\section{Medicinal \& Analytical Chemistry International Journal}

antigens, peptide hormones, neuropeptides, peptide antibiotic, and peptide based toxins are desirable. However, due to the poor pharmacokinetic properties and poor enzymatic stability, natural L- $\alpha$-amino acids in peptide sequences are often substituted with unnatural amino acids such as D- $\alpha$-amino acid and $\omega$-amino acid. Peptidoglycans such as $N$-acetylmuramic acid (NAM) and $\mathrm{N}$-acetyl glucosamine (NAG) derivatives are responsible for the development of bacterial cell wall. Vancomycin is a glycopeptides antibiotic that binds selectively to a D-AlaD-Ala dipeptide moiety of the NAM and NAG derivative through hydrogen bonds and prevents cell wall synthesis and hence destructs the bacterial cell [4].

Oligonucleotides have a noteworthy benefit because the binding mode of the receptor may be tailored using in vitro choice manner by means of biotechnology. Aptamerbased therapeutics for cancer treatment has revealed promise in clinical trials [5]. The pharmaceutical accomplishment of aptamers is due to the selective binding with a specific biological molecule such as protein surface. Aptamers can be covalently modified with imaging agents, chemotherapeutic agents etc., for theranostic applications. The first US Food and Drug Administration (FDA) approved aptamer drug is Macugen (pegaptanib), which is used to cure the age-related macular degeneration.

Appropriate knowledge of biology and chemistry of tumor site may be useful to control the growth of various tumors. Endothelial gap in tumor site is much more with respect to normal cell which is known as leaky vasculature. Liposome nanocarriers (100-200 nm) are permeable through this leaky vasculature of tumor site compared to normal cell and due to poor lymphatic drainage it retains at the tumor site. It is known as enhanced permeability and retention (EPR) effect and it is a passive targeting to tumor [6]. Moreover, a number of receptors are overexpressed on the tumor site such as epidermal growth factor receptor, G protein-coupled receptors, folate receptor etc. Active tumor targeting may be accomplished by functionalizing outer membrane of the liposome with suitable ligands such as folic acid based ligands, peptide-based ligands etc. It may promote specific cancer cell targeting and binding though molecular recognition. Doxil (pegylated nanoliposome encapsulated with doxorubicin) is the first FDA approved liposome-based anticancer nano-drug. Aberrant levels of carbohydrates are expressed by cancer cell known as tumor-associated carbohydrate antigens (TACAs) [7]. TACAs are very important for the progress of carbohydrate-based cancer vaccine and cancer immunotherapy. Moreover, tumor microenvironment (cytosol) is slightly acidic and usually has a $\mathrm{pH}$ of 6.5-7.2, endosomal $\mathrm{pH}$ 5.0-6.0 and lysosomal $\mathrm{pH}$ value 4.0-5.0, which are significantly different than the physiological $\mathrm{pH}$ 7.4. So, acidic $\mathrm{pH}$ activated drug design can be a promising strategy for selective targeting of tumor. It is essential to develop some probe that can differentiate cancer cells from normal cells which can be useful for the early detection of tumors.

Design of drug molecules conjugated with targeting moiety and imaging agent for specific binding to cellular organelles such as mitochondria is highly desirable for organelle selective theranostic applications. It may assist to gain key information about the organelle and their function in cellular processes, like cellular differentiation, signaling, and apoptosis. This knowledge may be exploited in various practical applications, including image guided cancer surgery, targeted drug delivery etc.

Chemistry plays an important role to explain the structure and function of biological molecular machinery such as membrane fusion machinery, RNA polymerase, kinesin walker, F1F0 ATP synthase, ribosome etc [8]. Membrane fusion is a necessary process in life and plays a decisive role in exocytosis, endocytosis, cellular trafficking, fertilization, viral infection, organelle formation, hormone secretion, and neurotransmitter release. Neuronal membrane fusion is directed by precise structure of proteins, such as SNARE [Soluble NSF (Nethylmaleimide-sensitive factor) Attachment Protein Receptor] [9]. Molecular recognition at the lipid membrane-water interface as well as TMD/TMD recognition play central role in SNARE protein induced membrane fusion. However, the particular mechanism of membrane fusion is still debated. Design and construction of artificial SNAREs may help to inspect the mechanism of biological membrane fusion. Moreover, protein, nucleic acid, neurotransmitter, hormone, and drug release to a target cell at the right time in a precise manner can be a promising approach through synthetic membrane fusion. The biological molecular machines such as protein motors kinesin and dynein are responsible for the contraction of muscle fibers or transportation of biological cargo around cells using the chemical energy stored in ATP, the chemical fuel of biological systems. Synthetic molecular machinery is required to understand the mechanism and function of each important fragment in the complex biological machinery.

Better advancement in medicinal chemistry can be achievable using proper design and methodology in organic synthesis, suitable analytical techniques and instrumentation, and advanced computational chemistry. 


\section{Medicinal \& Analytical Chemistry International Journal}

There is a need for collaboration between chemist, biologist, computational experts, bioinformaticians, and clinicians to tackle some of the grand challenges of the drug design and development process. Their collective efforts may result in the development of effective drug discovery with higher productivity, lower cost, and higher rate of success.

\section{References}

1. Mager MD, LaPointe V, Stevens MM (2011) Exploring and exploiting chemistry at the cell surface. Nat Chem 3(8): 582-589.

2. Müller C, Despras G, Lindhorst TK (2016) Organizing multivalency in carbohydrate recognition. Chem Soc Rev 45(11): 3275-3302.

3. Li H, Pesce E, Sheppard DN, Singh AK, Pedemonte N (2018) Therapeutic approaches to CFTR dysfunction: From discovery to drug development. J Cystic Fibrosis 17(2S): S14-S21.
4. Kahne D, Leimkuhler C, Lu W, Walsh C (2005) Glycopeptide and Lipoglycopeptide Antibiotics. Chem Rev 105(2): 425-448.

5. Zhou J, Rossi J (2017) Aptamers as targeted therapeutics: current potential and challenges. Nat Rev Drug Discovery 16(3): 181-202.

6. Wilhelm S, Tavares AJ, Dai Q, Ohta S, Audet J, et al. (2016) Analysis of nanoparticle delivery to tumours. Nat Rev Mater 1(5): 16014-16026.

7. Seeberger PH, Werz DB (2005) Automated synthesis of oligosaccharides as a basis for drug discovery. Nat Rev Drug Discov 4(9): 751-763.

8. Schliwa M, Woehlke G (2003) Molecular motors. Nature 422(6933): 759-765.

9. Rothman JE (2014) The Principle of Membrane Fusion in the Cell (Nobel Lecture). Angew Chem Int Ed 53(47): 12676-12694. 